\title{
Some Integrals of the Arctangent Function
}

\author{
By M. L. Glasser
}

Integrals of the form $\int_{0}^{\infty}\left(\tan ^{-1} c z\right)^{2 n} R(z) d z$, where $R(z)$ is an even rational expression in $z$, occur in the theory of localized magnetic moments in metals. Since integrals of this general type do not appear to be tabulated, we present here a method for evaluation as well as some interesting related material.

By partial fraction decomposition all integrals of the above type may be reduced to the form

$$
I_{n}(a)=\int_{0}^{\infty}\left(\tan ^{-1} c z\right)^{2 n}\left(z^{2}+a^{2}\right)^{-1} d z,
$$

where $a$ is not required to be real. Also, by a simple change of variable, only the case $c=1$ need be considered. By writing this as half the integral from $-\infty$ to $\infty$ and making the substitution $z=\tan \theta / 2$ this may be brought into the form $2^{-(2 n+1)}\left(a^{2}+1\right)^{-2} \int_{-\pi}^{\pi} \theta^{2 n}(1+\lambda \cos \theta)^{-1} d \theta$, where $\lambda=\left(a^{2}-1\right) /\left(a^{2}+1\right)$. In terms of $z=e^{i \theta}$, this may be written

$$
I_{n}(a)=(-1)^{n+1} i 2^{-2 n}\left(a^{2}-1\right)^{-1} \int_{\Gamma_{1}}\left(z-z_{0}\right)^{-1}\left(z-z_{1}\right)^{-1} \ln ^{2 n} z d z
$$

where $\Gamma_{1}$ is the contour $z=e^{i \theta},-\pi<\theta<\pi$ and $z_{0}=1 / z_{1}=(1-a) /(1+a)$. For the moment we assume $0<a<1$ so $z_{0}>0$ and lies inside the unit circle. Closing $\Gamma_{1}$ by the loop $\Gamma_{2}: z=\rho e^{ \pm i \pi}, 0<\rho<1$, we trap the pole at $z_{0}$ and thus

$$
I_{n}(a)=\frac{(-1)^{n} 2 \pi}{2^{2 n}\left(a^{2}-1\right)}\left\{\frac{\ln ^{2 n} z_{0}}{\left(z_{0}-z_{1}\right)}+\frac{1}{2 \pi i} \int_{\Gamma_{2}} \frac{\ln ^{2 n} z d z}{\left(z-z_{0}\right)\left(z-z_{1}\right)}\right\} .
$$

The integral remaining in (3) is

$$
J_{n}=\frac{1}{2 \pi i} \int_{0}^{1} \frac{(\ln x-i \pi)^{2 n}-(\ln x+i \pi)^{2 n}}{\left(x+z_{0}\right)\left(x+z_{1}\right)} d z
$$

This can be reduced to a sum of Kummer's Lambda functions [1]

$$
\Lambda_{n+1}(x)=\int_{0}^{x} \frac{\ln ^{n}|u|}{1+u} d u
$$

for example. However, since the resulting formula is somewhat unwieldy, the method will be illustrated by the cases $n=1,2$. We have

$$
\begin{aligned}
J_{1} & =-2\left(z_{1}-z_{0}\right)^{-1} \int_{0}^{1} \ln x\left\{\left(x-z_{0}\right)^{-1}-\left(x+z_{1}\right)^{-1}\right\} d x \\
& =\frac{2 z_{0}}{z_{0}{ }^{2}-1}\left\{(1 / 2) \ln z_{0} \ln \left[\frac{\left(1+z_{0}\right)^{2}}{z_{0}}\right]+\operatorname{Li}_{2}\left(\frac{z_{0}}{z_{0}+1}\right)-\operatorname{Li}_{2}\left(\frac{1}{z_{0}+1}\right)\right\}
\end{aligned}
$$

Received July 26, 1967. 
where $\mathrm{Li}_{2}(x)$ is the Euler dilogarithm [1]. Using the relation $\mathrm{Li}_{2}(x)+\mathrm{Li}_{2}(1-x)$ $=\pi^{2} / 6-\log x \log (1-x)$, we find

$$
I_{1}(a)=(\pi / 4 a)\left\{\frac{\pi^{2}}{6}-\ln ^{2}\left(\frac{1+a}{2}\right)-2 \operatorname{Li}_{2}\left(\frac{1-a}{2}\right)\right\} .
$$

Since both sides of this equation are analytic functions of $a$ for $\operatorname{Re} a>0$, the result is valid for all positive $a$. In addition, taking the limit $a \rightarrow 0$ leads to the known result $I_{1}(0)=\pi \ln 2$. The dilogarithm can be evaluated in closed form for a number of special cases [1], which leads to the apparently new results

$$
\begin{aligned}
I_{1}(\sqrt{ } 5) & =(\pi / 4 \sqrt{ } 5)\left\{\left(3 \pi^{2} / 10\right)-2 \ln ^{2}\left(\frac{1+\sqrt{ } 5}{2}\right)\right\}, \\
I_{1}(3) & =(\pi / 12)\left\{\left(\pi^{2} / 3\right)-\ln ^{2} 2\right\}, \\
I_{1}(\sqrt{ } 5-2) & =\frac{\pi}{4(\sqrt{ } 5-2)}\left\{\frac{\pi^{2}}{30}+\ln ^{2}\left(\frac{\sqrt{ } 5+1}{2}\right)\right\}, \\
I_{1}(\sqrt{ } 5+2) & =\frac{\pi}{4(\sqrt{ } 5+2)}\left\{\frac{11 \pi^{2}}{30}-5 \ln ^{2}\left(\frac{1+\sqrt{ } 5}{2}\right)\right\} .
\end{aligned}
$$

Other than for the trivial case $a=1$, these are the only real values of $a$ for which $I_{1}(a)$ may be expressed in elementary terms. The derivative of $I_{1}(a)$ is related to entry 3.813(5) of Gradshteyn and Ryzhik's tables [2] so (7) could also be obtained from that result by integration.

The case $n=2$ leads to

$$
\int_{0}^{\infty} \frac{\left(\tan ^{-1} z\right)^{4}}{z^{2}+a^{2}} d z=(\pi / 4 a)\left\{\frac{\pi^{4}}{40}+\pi^{2} \operatorname{Li}_{2}\left(\frac{a-1}{a+1}\right)-6 \operatorname{Li}_{4}\left(\frac{a-1}{a+1}\right)\right\}
$$

in terms of tabulated functions [1]. Unfortunately, the tetralogarithm cannot be evaluated in closed form for many special values. The case $a \rightarrow 0$ leads to

$$
\int_{0}^{\pi / 2} x^{4} \csc ^{2} x d x=\frac{\pi^{3}}{2} \ln 2-\frac{9 \pi}{4} \zeta(3) .
$$

This integral can be obtained from [2, Eq. 3.748.2, p. 418], which leads to

$$
\int_{0}^{\pi / 2} x^{p+1} \csc ^{2} x d x=(p+1)(\pi / 2)^{p}\left\{p^{-1}-2 \sum_{k=1}^{\infty}(p+2 k)^{-1} 2^{-2 k} \zeta(2 k)\right\}
$$

in terms of the Riemann Zeta function. Thus, for $p=3$ we have the interesting relation

$$
\zeta(3)=\frac{4 \pi^{2}}{9}\left\{\sum_{k=1}^{\infty} \zeta(2 k) /\left(4^{k}(2 k+3)\right)+\frac{1}{2} \ln 2-\frac{1}{6}\right\} .
$$

In a similar way we can sum the series $\sum_{k=1}^{\infty} \zeta(2 k) 2^{-2 k} /(2 k+p)$ for any odd $p$.

The method used here can also be extended to arbitrary integrals of the form $\int_{0}^{\infty}\left(\tan ^{-1} z\right)^{n} R(z) d z$ where $n$ and $R$ are not required to be even. When symmetry is not invoked, however, the Cauchy principal part rather than the residue is involved.

Finally, it is emphasized that $a$ is not restricted to real values, so that cases such as $R(z)=\left(1+z^{6}\right)^{-1}$ may also be treated. The polylogarithms of complex 
argument have been studied in detail [1] and we also obtain results as

$$
\int_{0}^{\infty} \frac{\left(\tan ^{-1} z\right)^{2}}{\left(z^{2}-3\right)-4 i} d z=\frac{\pi(1+2 i)}{20}\left\{\frac{7 \pi^{2}}{48}-\frac{1}{4} \ln ^{2} 2+\frac{\pi \ln 2}{4}-2 i \beta(2)\right\}
$$

where $\beta(2)$ is Catalan's constant $0.915965 \cdots$. Taking the real and imaginary parts of both sides of (13) gives

$$
\begin{aligned}
& \int_{0}^{\infty} \frac{\left(\tan ^{-1} z\right)^{2}}{z^{4}-6 z^{2}+25} d z=\frac{\pi}{40}\left\{\frac{7 \pi^{2}}{48}-\frac{1}{4} \ln ^{2} 2+\frac{\pi \ln 2}{4}-\beta(2)\right\}, \\
& \int_{0}^{\infty} \frac{z^{2}\left(\tan ^{-1} z\right)^{2}}{z^{4}-6 z^{2}+25} d z=\frac{\pi}{8}\left\{\frac{7 \pi^{2}}{48}-\frac{1}{4} \ln ^{2} 2+\frac{\pi \ln 2}{4}+\beta(2)\right\} .
\end{aligned}
$$

These are only a few of the special cases which can be expressed in closed form.

Acknowledgement. The author wishes to thank Drs. V.E. Wood and R.P. Kenan for helpful comments.

\section{Battelle Memorial Institute}

Columbus, Ohio 43201

\#4264. I. S. Gradshteyn \& I. M. Ryzhik, Table of Integrals, Series and Products, translated from Russian, Academic Press, New York, 1965. MR 33 \#5952. 